\title{
Microwave Imaging Techniques for Biomedical Applications
}

\author{
A.Joise $1^{\circ}$, J.Mallorqui*, A.Broquetas*, J.M.Geffrin ${ }^{\circ}$, \\ N.Joachimowicz ${ }^{\circ}$, M.Vall.lossera*, L.Jofre*, J.Ch.Bolomey ${ }^{\circ}$ \\ 'Department of Electromagnetic Research \\ Supélec, Plateau de Moulon, 91192 Gif-sur-Yvette, France \\ * Department of Signal Theory and Communications, \\ Universitat Politecnica de Catalunya, Campus Nord-D3, 08034 Barcelona, Spain
}

\begin{abstract}
The specific properties of microwaves have been used for a while in many applications. More particularly, the penetration through opaque media makes microwaves a convenient agent for non invasive testing, evaluation and measurements. For example, industrial sensors have been developed, based on the sensitivity of microwave propagation constant upon quartities of practical relevance, such as water contents, temperature or composition. Similarly, microwaves have been considered for medical applications involving the detection of organ movements and changes in tissue water content [1]. More particularly cardiopulmonary interrogation via microwaves has resulted in various sensors for monitoring ventricular volume change or movement, arterial wall motion, respiratory movements, pulmonary oedema, etc [2]. In all these applications, microwave sensors perform local measurements and need to be displaced for obtaining an image reproducing the spatial variations of a given quantity. The effective starting of microwave imaging techniques for biomedical applications can be dated at the beginning of the 80's, with the pioneer contribution of Drs E.Larsen and J.Jacobi, from the Walter Reeds Army Institute. Using the water-immersion technique [3], the first transmission and tomographic images of biological targets, such as perfused organs, have been shown to offer promises thanks to their contrast and unexpected spatial resointion [4.5]. More recently, advances in the area of inverse scattering theory and microwave technology have made possible the development of microwave imaging and tomographic instruments. This paper provides a review of such equipments developed at Supelec and UPC Barcelona, within the frame of successive French-Spanish PICASSO cooperation programs. It reports the most significant results and gives some perspectives for future
\end{abstract}

developments. In a first part, a brief historical survey will be given. In the following, both technological and numerical aspects will be considered. The results of preliminary pre-clinical assessments and in-lab experiments will allow to illustrate the capabilities of the existing equipment, as well as its difficulty in dealing with clinical situations. Finally, some remarks on the expected development of microwave imaging techniques for biomedical applications will be given.

\section{Reconstruction algorithms}

The main difficulty in producing microwave images of quality was to compensate for complex diffraction mechanisms. Two classes of approaches have been, and are still, used. The first one tries to take profit of available efficient reconstruction algorithms used with $\mathrm{X}$-ray CT. X-rays propagate along linear paths, and using these algorithms in a diffraction situation requires to eliminate multipath contributions between the transmitter and the receiver. This has been achieved by using time spectrometry which allows, thanks to a convenient time windowing, to isolate the shortest path corresponding to almost straight line propagation $[6,7]$. To some extent, this first approach consists in a hardware attempt to compensate the effects of the scattering phenomena. In the second approach, on the contrary, the scattering mechanisms are formally taken into account in the reconstruction process, more or less rigorously. Indeed, two main approaches can be considered. The first one neglects multiple scattering in the target. This approach, imported from ultrasound imaging techniques [8], is known as diffraction tomography. Such a lowdiffraction assumption allows to linearize the inverse scattering problem, in that sense that the scattered field data are linearly related to the equivalent currents induced 
in the target by the interrogating beam. The reconstruction can be easily performed by using efficient Fast Fourier Transform algorithms, which can be viewed as a generalization of Fourier transform algorithms used with X-ray CT. Consequently, this approach is very efficient under the computational aspect and is particularly well suited for real time operations. However, the spectral processing neglects the evanescent part of the scattered field, and, as a result, the spatial resolution is limited to one half wavelength. Furthermore, this approach does not allow for quantitative imaging, because the reconstructed induced currents depend both on the local properties of tissues and on the total local field which varies within the target. The images obtained with diffraction tomography algorithms only reproduce the shapes of structures exhibiting low dielectric contrast, as for ultrasound echotomography in the case of soft tissues. The second approach, on the contrary, is able to provide quantitative images, even for high contrast targets. The images can be calibrated in terms of complex permittivity. With respect to previous diffraction tomography, the price to pay is very significant at the computation level. Indeed, an exact inversion of the scattering problem needs to iteratively solve a non-linear equation for the dielectric contrast. Figure 1 shows a flow chart of the iterative reconstruction algorithm.

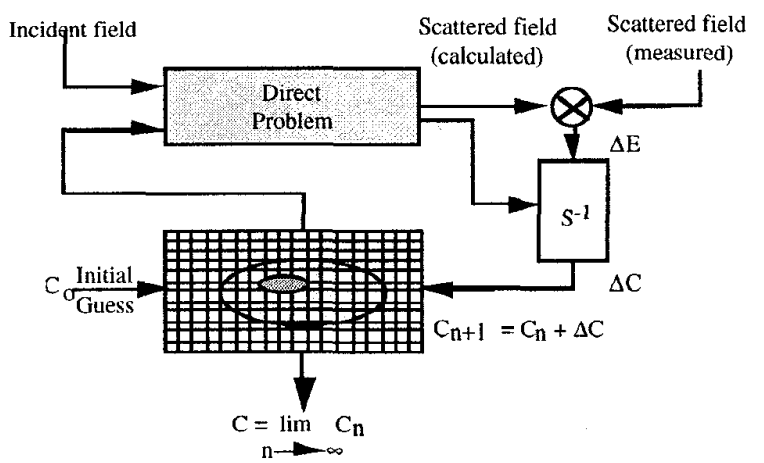

Figure 1: Principle of the quantitative reconstruction of the dielectric contrast by means of a non-linear iterative technique

At each step of the iterative process, the measured scattered field is compared to the scattered field calculated from a numerical model. The permittivity contrast of the model is progressively adjusted by minimizing the error between measured and calculated scattered fields. To this end, the permittivity contrast is updated by inverting an ill-conditioned linearized sensitivity matrix relating the contrast update to the scattered field error. Such an approach, equivalently known as Distorted Born Method
(DBM) [9] or Newton Kantorovitch Technique (NKT) [10], has been developped at the end of the 80's and has been shown to be able to deal with high contrasted structures. The computational load does not allow yet for real time reconstruction, however, parallel computers are expected to significantly improve the situation from this aspect [11]. An interesting feature of iterative techniques is that the spatial resolution is not so strictly related to the wavelength, as it is for diffraction tomography, just because evanescent waves are fully included in the formulation. Consequently, spatial resolutions comprised between one fifth and one tenth of the wavelength are possible to obtain, according to the available experimental and numerical signal to noise ratios.

\section{Equipment}

Two kinds of equipment for biomedical applications have been considered, differing from the geometry of the transmitter/receiver arrangement. Both have been designed for operation according to a transmission mode in water, at a frequency of the order of $2 \mathrm{GHz}$. Such a frequency provides a satisfactory optimization between a desired investigation depth of about $20 \mathrm{~cm}$, and a spatial resolution comprised between 5 and $10 \mathrm{~mm}$. The first one, developped at Supelec, consists of a planar microwave camera operating at $2.45 \mathrm{GHz}$ [12] (Figure 2).

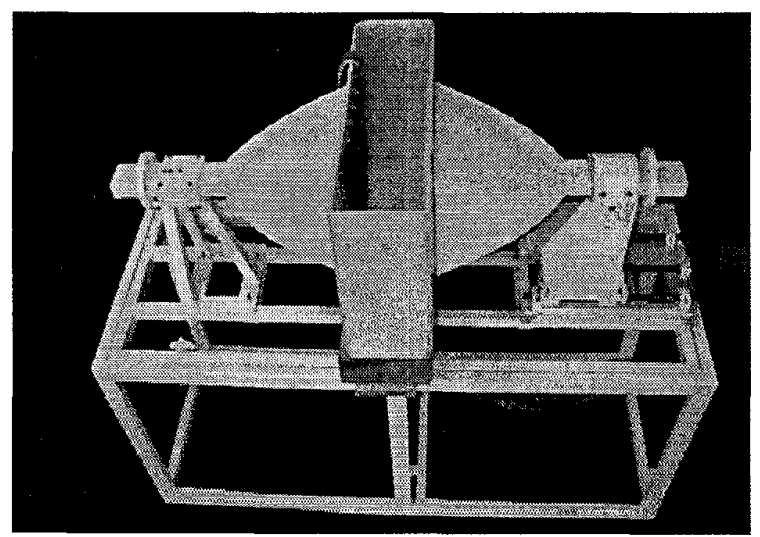

Figure 2: Planar microwave camera

The receiving antenna array covers a square surface of 22 by $22 \mathrm{~cm}$. The biological target, immersed in water, is illuminated by a microwave collimated beam produced by a lens-corrected horn and can be rotated around a vertical axis to obtain multiviewing. The field scattered by the target is measured by means of an array of $32 \times 32=1,024$ short dipoles loaded by PIN diodes and operated according to the modulated scattering technique. Until the beginning of 1998 , the acquisition time for a projection was equal to 
$1 \mathrm{~s}$. As explained later, a time reduction by a factor 10 has been recently obtained. The second equipment, developped at UPC Barcelona, is a circular scanner operating at $2.33 \mathrm{GHz}$ [13] (Figure 3).

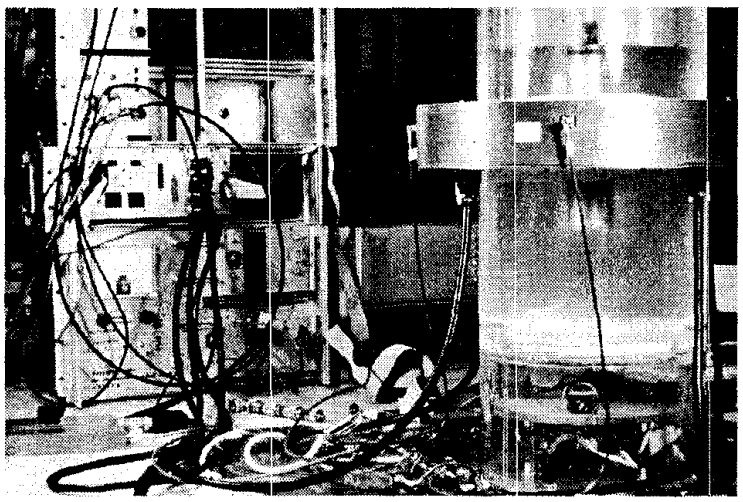

Figure 3: Circular microwave scanner

The Transmit/Receive array consists of 64 multiplexed horns distributed on a $25 \mathrm{~cm}$ radius circle. When one of these horns is transmitting, the 33 opposite horns are used for reception. The acquisition time is about $3 \mathrm{~s}$.

\section{Results}

The microwave camera has been initially designed for non-invasive thermometry (NIT) purposes during hyperthermia sessions $[14,15]$. The idea was to take profit from the significant temperature sensitivity of the dielectric constant in order to image deep temperature gradients [16]. Indeed, hyperthermia is demanding for an accurate control of the temperature distribution in the heated tissues. Such a control is impossible to obtain in case of deep seated tumours, except with invasive thermometric probes. This explain the until now open area for non-invasive thermometry, where microwaves has mainly to compete with NMR imaging techniques. By the way, retrieving the temperature from the dielectric constant is not so easy, due to the non-stationary aspect of living tissues. The temperature coefficient depends not only directly on temperature, but also on thermal induced thermoregulatory processes. Preliminary experiments conducted on phantoms, isolated organs and volunteers $[17,18]$ have confirmed the significant sensitivity of microwave images with respect to temperature and blood flow rate. Some pre-clinical assessments have been engaged in various applications involving thermal effects or vasculatory damages. This has been the case for inflammatory processes resulting from organ rejection after transplant, and for fibrosis after therapeutic or accidental irradiation. The high sensitivity to water content has led to investigate the monitoring and localization of lung water content. In all these trials, microwave images have been shown to be non completely compatible with clinical requirements, for both insufficiently accurate reconstruction via diffraction tomography, and for experimental difficulties, related for instance to the need for waterbolluses. Furthermore, the time required for reconstructing images was too long for obtaining interactivity between operator and equipment. However, very recently, the microwave camera has been made compatible with real time constraints. Images can now be produced at the rate of more than 10 images per second, which is enough for most biological processes. Such real time capability has suddenly renewed the interest for this imaging equipment which is particularly well suited for gaining experience in observing dynamically interactions of living structures with microwave beams. For instance, images such as this given in Figure 4 can be observed in real time, while the hand is moved. A video tape has been prepared for this purpose.

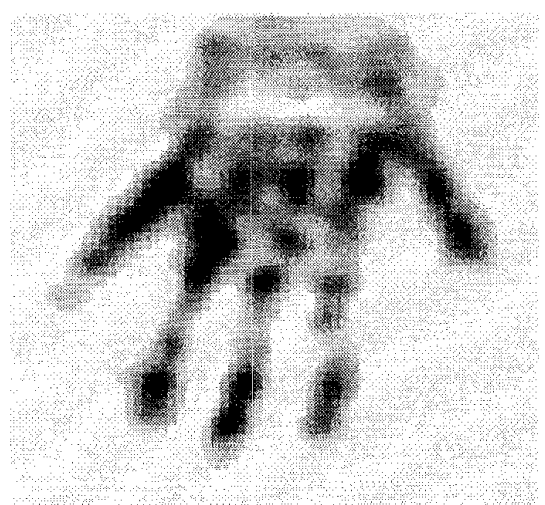

Figure 4: Microwave image of a human

hand. Mono-view reconstruction with a diffraction tomography algorithm

The microwave scanner has been developped for the objectives which have been already described [19,20,21], but in addition to brain imaging [18]. Many. investigations have been conducted on phantoms and volunteers. Initially, the reconstructions were performed by using diffraction tomography algorithms, with the difficulties associated to imaging biological structures with high dielectric contrast. Fortunately, the development of iterative reconstruction algorithms has open the way to quantitative imaging. The data obtained a few years before have been successfully processed for retrieving the complex permittivity of a human fore-arm $[10]$. For the sake of comparison, Figure 5 allows to compare the results obtained from the same set of 
experimental data with i) diffraction tomography and ii) an iterative approach.
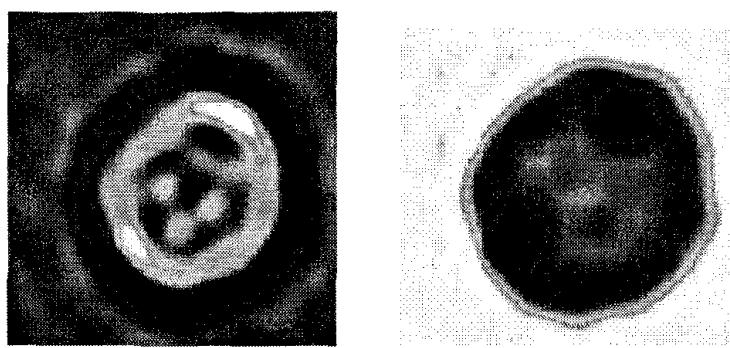

Figure 5: Human fore-arm reconstructed from the microwave scanner data: linear diffraction tomography (left) and non-linear iterative technique (right).

It can be seen that diffraction tomography just provides the external contour of the arm but does not provide any information on the arm inner structure. On the contrary, the image obtained from the iterative algorithm clearly shows the bone and muscle structures, with image levels corresponding to the expected dielectric contrast for these tissues. It is worth noting that diffraction tomography processing provides the external contour of the target which can be efficiently used for initiating the iterative process. Extensive trials with calibrated samples of known permittivity have allowed to confirm the robustness of the reconstruction process with respect to noise and its stability versus various parameters of experimental or numerical orders, especially versus the a priori information [22]. One major difficulty in using this rigorous approach is to reduce as much as possible the model noise, by using a numerical model reproducing as closely as possible the configuration of the experimental setup.

\section{Perspectives}

The experience gained with the $2.45 \mathrm{GHz}$ planar camera and the $2.33 \mathrm{GHz}$ scanner, as well as the recent advances in iterative reconstruction techniques have led to consider two distinct ways of developing the microwave imaging activities at Supelec and UPC Barcelona. The first one aims to still increase and exploit the real time capabilities offered by the planar microwave camera. Actually, the most time constraining task is the acquisition of the scattered field data. This constraint will be drastically reduced by considering a parallel, instead of a sequential, modulation scheme of the $32 \times 32$ antenna array. As a result, the acquisition time will be reduced by factor 1,000 , or less but with a better signal to noise ratio. In addition, a new transmitting antenna will be re- designed to improve the uniformity of the field distribution in the microwave interrogation beam and to allow to operate with two orthogonal polarizations. Such a polarization diversity will significantly contribute to improve the image quality and the interpretation capability of microwave images.

The second trail to be followed is the development of a new generation of microwave scanner, fitting as much as possible contradictory requirements such as improving the image quality while decreasing the equipment/algorithm complexity. Designed for whole body operation, the scanner consists of a $60 \mathrm{~cm}$ diameter circular array of 64 printed, H-type, antennas (Figure 6).

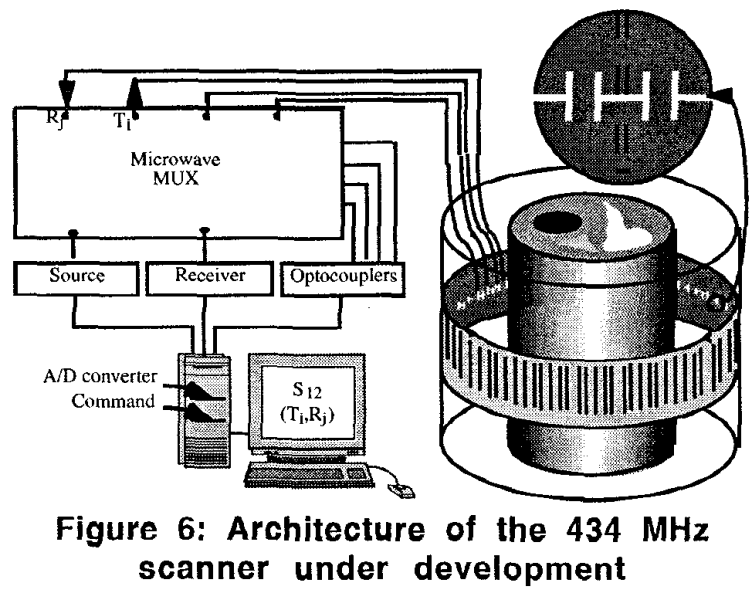

Such a fixed antenna array is expected to drastically improve the results already obtained with an early version of scanner, using mechanical movements of transmitting and receiving antennas [23]. The new spatial resolution capabilities of iterative techniques have impacted the choice of the operating frequency, which has been decreased down to $434 \mathrm{MHz}$. Decreasing the frequency reduces the propagation losses in water and tissues, and allows to perform some oversampling with respect to standard sampling rules. One new difficulty resulting from the decrease of the frequency is that the scanner environment must be more carefully taken into account. More particularly, the array of antennas and its supporting frame become more visible. While first reconstructions from the $2.33 \mathrm{GHz}$ microwave scanner data were performed in "free-space", it is no longer possible, at $434 \mathrm{MHz}$, to neglect the scanner boundaries for a $60 \mathrm{~cm}$ diameter whole-body configuration. The reconstruction process is more complicated than in free-space [24,25]. The development of this new scanner will take profit of the computation power reinforcement resulting from the use of a parallel computer. Systematic investigations have been conducted at the European Centre for 
Parallelism of Barcelona (CEPBA) which is equipped with a SGI O2000 of 64 MIPS R10000 processors and has a theoretical peak performance of $25 \mathrm{Gflops} / \mathrm{s}$. Such an efficient computation tool is currently used for optimizing the equipment performance/complexity ratio. For instance, Figure 7 shows how the image quality is changing when less and less receiving antennas are effectively used in the reconstruction process.

\section{Conclusion}

From the beginning of the 80 's, microwave imaging techniques for biomedical applications have been drastically improved. It can be observed that an increasing number of research groups are devoting their activity to this more promising than ever topic [e.g.26,27,28,29,7]. Various assessments conducted with preliminary equipments have confirmed the sensitivity of microwave images to factors of medical relevance. Even if operational and clinical efficacy is not yet achieved, at least the different steps for succeeding are now well identified. The solutions are almost already to hand, and the problem is undoubtedly more of financial than technical order. Indeed, without a significant development effort, microwave irnaging techniques will still have to wait a long time before being recognized and accepted by their potential users.

\section{Acknowledgement}

The cooperation between the French and the Sparish groups has been undertaken within Picasso Integrated Action Programs. The Spanish codes were run in vectorial computers operated by the European Centre for Parallelism of Barcelona (CEPBA)

\section{References}

[1] Medical Applications of Microwave Imaging, L.E.Larsen \& J.H.Jacobi Eds., pp.47-58, IEEE Press, New York, 1986

[2] J.C.Lin, "Microwave propagation in biological dielectrics with application to cardiopulmonary interrogation", in Medical Applications of Microwave Imaging, L.E.Larsen \& J.H.Jacobi Eds., pp.47-58, IEEE Press, New York, 1986

[3] J.H.Jacobi, L.E.Larsen, "Water immersed microwave antennas and their aplication to microwave interrogation of biological targets", IEEE Trans, Theory Tech., MTT-27. pp.70-78, 1979

[4] L.E.Larsen, J.H.Jacobi, "Microwave scattering parameters imasery of isolated carine kidney", IEEE Trans.Nucl.Sci., NS-27, pp.1184-1 191, 1980
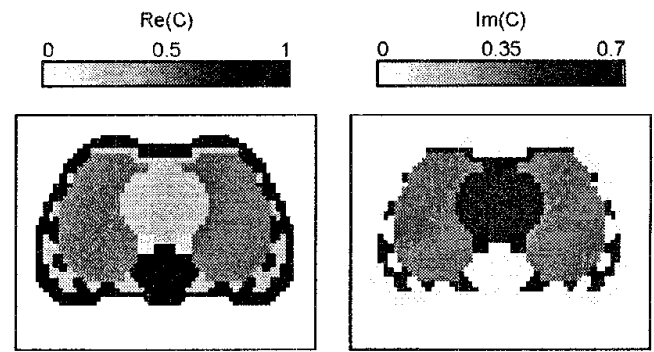

Thorax model, mesh $39 \times 51$

Re and Im parts of the dielectric contrast $C$
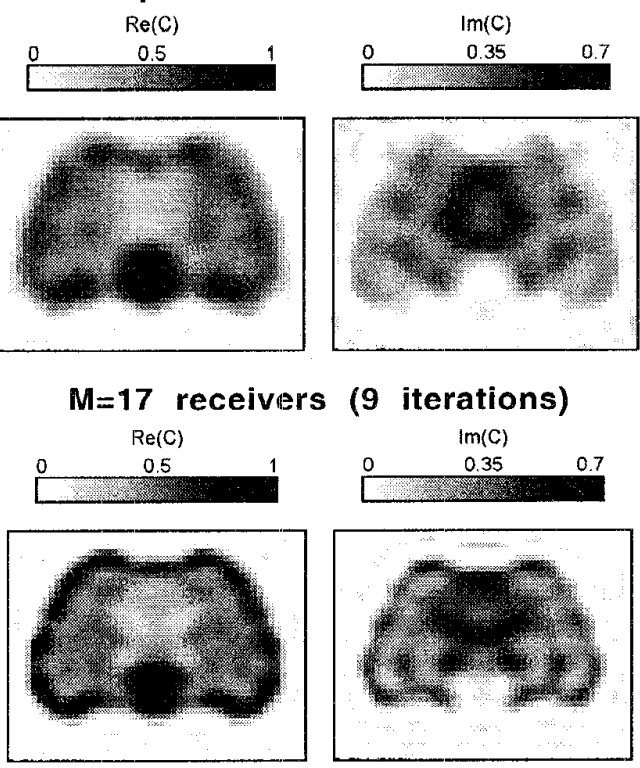

$M=25$ receivers

$\operatorname{Re}(\mathrm{C})$

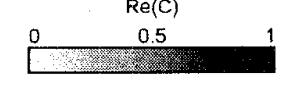

(10 iterations)
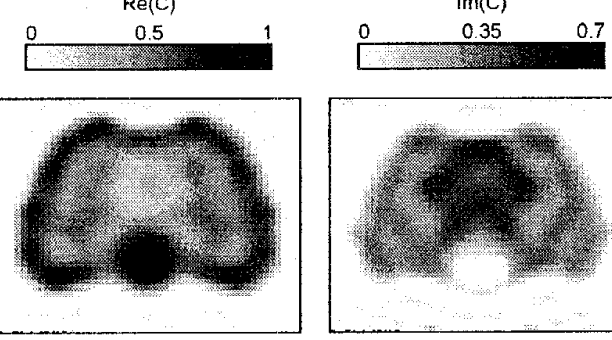

$M=32$ receivers (14 iterations)

Figure 7: Effect of the number of receiving antennas of a circular scanner

Freqency: $434 \mathrm{MHz}$; Diameter: $60 \mathrm{~cm}$; Nb. of antennas: 32

Number of receiving antennas effectively used in the reconstruction: $M$. 
[5] L.E.Larsen, J.H.Jacobi, "Microwaves offer promise as imaging modality", Diagn.Imag. Clin. Med., vol.11, pp. 44-47,1982

[6] J.H.Jacobi, L.E.Larsen, "Microwave time delay spectroscopy imagery of isolated canine kidney", Med.Phys.,7, pp.1-7, 1980

[7] M.Miyakawa, "Tomographic imaging of temperature change in a phantom of human body using a chirp radar type microwave tomograph", Med.Biol.Eng.Comput., 29, 2:745-752, 1991

[8] R.K.Mueller, M.Kaveh, G.Wade, "Reconstructive tomography and applications to ultrasonics", Proc.IEEE, vol.67, pp.567-587, 1979

[9] W.C.Chew, Y.M.Wang, "Reconstruction of twodimensional permittivity distribution using the distorted Born iterative method", IEEE Trans. Med. Imag., vol.9, 218,1990

[10] N.Joachimowicz, Ch.Pichot, J.P.Hugonin, "Inverse scattering: an iterative method for electromagnetic imaging", IEEE Trans. Antennas Propagat., vol.AP-39, $\mathrm{n}^{\circ} 12$, pp.1742-1752, 1991

[11] J.Mallorqui, O.Cabalero, A.Broquetas, N.Joachimowicz, J.Ch.Bolomey, "Parallelisation of a Newton-Kantorovich reconstruction algorithm for microwave tomography", PIERS'98, 13-17 July, 1998, Nantes, p.1017

[12] J.Ch.Bolomey, Ch.Pichot, G.Gaboriaud, "Planar microwave imaging camera for biomedical applications: critical and prospective analysis of reconstruction algorithms", Radio Sc., vol.26, pp.541549, 1991

[13] A.Broquetas, J.Romeu, J.M.Rius, A.R.Elias-Fusté, A.Cardama, L.Jofre, "Cylindrical geometry: a further step in active microwave tomography", IEEE Trans. Microwave Theory Techn., vol. MTT-19, p.836-844, May 1991

[14] J.Ch.Bolomey, M.Hawley, "Non-invasive control of hyperthermia", in Gautherie M. Ed., Methods of hyperthermia control, Clinical Thermology Subseries Thermotherapy, Springer, Berlin, pp.35-111

[15] J.Ch.Bolomey, D.Le Bihan, M.Miyakawa, Recent trends in non-invasive thermal control", in ThermoRadiotherapy and -Chemotherapy, M.H.Seegenschmeidt et al. Eds, Chap.16, 361-379, Springer, Berlin, 1995

[16] J.Ch.Bolomey, L.Jofre, G.Peronnet, On the possible use of microwave active imaging for remote thermal sensing", IEEE Trans. Microwave Theory Techn., MTT$31,777,1983$

[17] Guerquin-Kern J.L., M.Gautherie, G.Peronnet, L.Jofre, J.Ch.Bolomey, "Active microwave tomographic imaging of isolated perfused animal organs, Bioelectromagnetics, 6, 145, 1985

[18] H.Almirall, A.Broquetas, L.Jofre, "Active microwavecomputed brain tomography: the response to a challenge", J.Neurosci. Methods, 36, 239, 1991

[19] J.M.Rius, Ch.Pichot, L.Jofre, J.Ch.Bolomey, N.Joachimowicz, A.Broquetas, M.Ferrando, "Planar and cylindrical active temperature imaging", IEEE Trans. Med.Imag., vol.11, pp.457-469, 1992

[20] J.Mallorqui, A.Broquetas, L.Jofre, A.Cardama, "Noninvasive active thermometry with a microwavetomographic scanner in hyperthermia treatments", Applied computational electromagnetics Society Journ., 7(2), pp.121-127, 1992

[21] L.Jofre, M.S.Hawley, A.Broquetas, E. de Los Reyes, M.Ferrando, J.Romeu, A.R.Elias-Fusté, "Medical imaging with a microwave tomographic scanner", IEEE Trans. Biom.Imag. vol.32, pp.303-312, Mar.1990

[22] N.Joachimowicz, J.Mallorqui, J.Ch.Bolomey, A.Broquetas, "Convergence and stability assessment of Newton-Kantorovich reconstruction algorithm for microwave tomography", accepted for publication in IEEE Trans. on Medical Imaging

[23] A.Joisel, J.M.Geffrin, J.M.Elissalt, K.Belkebir, N.Joachimowicz, J.Ch.Bolomey, "Iterative 2D reconstruction algorithms for microwave imaging systems", IEE Colloquium: The applications of microwave in medicine, London

[24] A.G.Tijhuis, K.Belkébir, A.Litman, J.M.Geffrin, J.Ch.Bolomey, "Two-dimensional inverse profiling: non-linear optimization and embedding", Proc.URSI Int.Symp. on Electromagnetic Theory, Thessaloniki, Greece, 25-28 May, 1998, pp.503-505

[25] O.Franza, N.Joachimowicz, J.Ch.Bolomey, "Formal compensation of sensor related interactions for quantitative microwave tomography", PIERS'98 Symp., 13-17 July, 1998, Nantes, France, p.450

[26] J.Ch.Bolomey, A.Joisel, "Microwave scanners for biomedical applications", in Proc.Microwave and RF'95 Conf., London, Oct.10-12, 1995, pp.209-214

[27] S.Caorsi, G.L.Gragnani, M.Pastorino, "Equivalent current density reconstruction for microwave imaging purposes", IEEE Trans. Medical Imaging, MI-12, 2, June 1993

[28] S.Semenov et al., "Microwave tomography. Two dimensional system for biological imaging", IEEE Trans. BME,

[29] P.M.Meaney, K.D.Paulsen, A.Hartov, R.K.Crane, "Active microwave imaging system for reconstruction of 2-D electrical property distribution", IEEE Trans. Biomed.Engineering, BME-42, 10, 1017-1026, Oct. 1995 